Currently the most distant galaxy cluster in which an Fe K-line has been detected is at a redshift of $z=0.54$ (ref. 18). Therefore, AX J2019 + 1127 is now the highest redshift cluster for which the existence of a metal-enriched intra-cluster medium has been confirmed. The detection of the large iron content at high redshift sets a new limit for the epoch of the enrichment. This favours models where early star burst phases in galaxies are responsible for the metal enrichment of the intra-cluster medium ${ }^{19,20}$.

More surprisingly, a high iron abundance has been discovered in this 'dark cluster', which is very poor in its galaxy content according to the deep optical searches ${ }^{3,4}$. The main optical light source from this cluster is galaxy $\mathrm{D}$ which has a blue luminosity of $L_{B}=1.1 \times 10^{11} h_{50}^{-2} L_{\odot}$ (where $L_{\odot}$ is the solar luminosity). Another possible member galaxy is galaxy $\mathrm{C}$ (ref. 1) which, however, can contribute only a fraction of the optical luminosity of galaxy D. Therefore, the mass-to-light ratio of the cluster is $M / L_{B} \approx 3,300(k T / 8.6 \mathrm{keV})^{-1} h_{50} M_{\odot} / L_{\odot}$. Because, according to our current understanding, the metal enrichment originates in the stars of the cluster galaxies, it is very puzzling that we detect such a high iron abundance in this 'dark cluster'. Therefore, either AXJ2019 + 1127 is a very new and enigmatic object or it has more cluster members at fainter magnitudes that have escaped the deep searches conducted so far. Recently, it has been reported ${ }^{21}$ that there are also some low-redshift clusters that are optically dark but bright in X-rays. Combined with our results, this may indicate that there exist many such objects in our Universe which have eluded optical identification. In any case, AX J2019 + 1127 provides us with a new means for testing cosmological theory and ought to be well studied in various wavelength bands.

Received 6 December 1996; accepted 21 May 1997.

1. Lawrence, C. R. et al. Discovery of a new gravitational lens system. Science 223, 46-49 (1984)

2. Lawrence, C. R. in Astrophysical Applications of Gravitational Lensing (eds Kochanek, C. S. \& Hewitt, J. N.) 299-304 (Springer, Berlin, 1995).

3. Schneider, D. P. et al. Deep optical and radio observations of the gravitational lens system $2016+112$. Astrophys. J. 294, 66-69 (1985).

4. Langston, G. I., Fischer, J. \& Aspin, C. Infrared $K$-band observations of the gravitational lens 2016+112. Astron. J. 102, 1253-1257 (1991).

5. Narasimha, D., Subramanian, K. \& Chitre, S. M. The gravitational lens system $2016+112$ revised. Astrophys. J. 315, 434-439 (1987).

6. Schneider, D. P. et al. The third image, the redshift of the lens, and new components of the gravitational lens $2016+112$. Astron. J. 91, 991-997 (1986).

Raymond, J. C. \& Smith, B. W. Soft X-ray spectrum of a hot plasma. Astrophys. J. Suppl. Ser. 35, 419 439 (1977).

8. Anders, E. \& Grevesse, N. Abundances of the elements-meteoritic and solar. Geochim. Cosmochim. Acta 53, 197-214 (1989)

9. Dickey, J. M. \& Lockman, F. J. HI in the Galaxy. Annu. Rev. Astron. Astrophys. 28, 215-261 (1990).

10. David, L., Slyz, A., Jones, C., Forman, W. \& Vrtilek, S. D. A catalog of intracluster gas temperatures. Astrophys. J. 412, 479-488 (1993).

11. Tsuru, T. et al. in The 11th Int. Colloq. on UV and X-ray Spectroscopy of Astrophysical and Laboratory Plasmas (eds Watanabe, T. \& Yamashita, K.) 375-378 (Universal Academy, Tokyo, 1996).

12. Williams, O. R. et al. The X-ray spectra of high-luminosity active galactic nuclei observed by GINGA Astrophys. J. 389, 157-178 (1992).

13. La Franca, F., Franceschini, A., Cristiani, S. \& Vio, R. On the relationship between the optical and Xray luminosities of quasars. Astron. Astrophys. 299, 19-24 (1995).

14. Gioia, I. M. \& Luppino, G. A. The EMSS Catalog of X-ray selected clusters of galaxies. I. An atlas of CCD images of 41 distant clusters. Astrophys. J. Suppl. Ser. 94, 583-614 (1994).

15. Luppino, G. A. \& Gioia, I. M. Constraints on cold dark matter theories from observations of massive X-ray-luminous clusters of galaxies at high redshift. Astrophys. J. 445, L77-L80 (1995).

16. Briel, U. G., Henry, J. P. \& Böhringer, H. Observation of the Coma cluster of galaxies with ROSAT during the All-Sky Survey. Astron. Astrophys. 259, L31-L34 (1992).

17. Ohashi, T. in Proc. 17th Texas Symp. on Relativistic Astrophysics and Cosmology (eds Böhringer, $\mathrm{H}_{\text {. }}$ Morfill, G. E. \& Trümper, J. E.) 217-220 (New York Acad. Sci., New York, 1995).

18. Donahue, M. Temperature and metallicity of a massive X-ray cluster at redshift 0.5. Astrophys. J. 468, 79-85 (1996).

19. Arnaud, M., Rothenflug, R., Boullade, O., Vigroux, L. \& Vangioni-Flam, E. Some constraints on the origin of the iron enriched intra-cluster medium. Astron. Astrophys. 254, 49-64 (1992).

20. Hattori, M. \& Terasawa, N. Metal enrichment of the intracluster medium from protogalaxies and the gamma-ray bursters. Astrophys. J. 406, L55-L58 (1993).

21. Ebeling, H., Mendes de Oliveira, C. \& White, D. A. A2572 and HCG94-galaxy clusters but not as we know them: an X-ray case study of optical misclassifications. Mon. Not. R. Astron. Soc. 277, 1006-1032 (1995).

22. Tanaka, Y., Inoue, H. \& Holt, S. S. The X-ray astronomy satellite ASCA. Publ. Astron. Soc. Jpn 46, L37L41 (1994).

23. Trümper, J. E. ROSAT. Phys. Scripta T7, 209-215 (1984).

24. Sarazin, C. L. X-ray emissions from clusters of galaxies. Rev. Mod. Phys. 58, 1-116 (1986).

Acknowledgements. We thank T. Sonobe, T. Miyaji, C. R. Lawrence, Y. Tanaka and M. Matsuoka for discussions. M.H. was supported in part by the post-doctoral program of the Max-Planck Gesellschaft and Yamada Science Foundation; Y.I. was supported by the Special Researchers' Basic Science Program of the Riken. H.B. and S.S. thank the Verbundforschung for support.

Correspondence should be addressed to M.H. (e-mail: hattori@astr.tohoku.ac.jp).

\section{Properties of ideal composite} knots

\section{Vsevolod Katritch, Wilma K. Olson^, Piotr Pieranski $\dagger$, Jacques Dubochet $\ddagger$ \& Andrzej Stasiak $\ddagger$}

* Department of Chemistry, Rutgers the State University of New Jersey, New Brunswick, New Jersey 08903, USA

$\dagger$ Institute of Physics, Poznan University of Technology, 60-965 Poznan and Institute of Molecular Physics, 60-159 Poznan, Poland

\$aboratoire d'Analyse Ultrastructurale, Bâtiment de Biologie,

Université de Lausanne, CH-1015 Lausanne-Dorigny, Switzerland

The shortest tube of constant diameter that can form a given knot represents the 'ideal' form of the knot $^{1,2}$. Ideal knots provide an irreducible representation of the knot, and they have some intriguing mathematical and physical features, including a direct correspondence with the time-averaged shapes of knotted DNA molecules in solution ${ }^{1,2}$. Here we describe the properties of ideal forms of composite knots-knots obtained by the sequential tying of two or more independent knots (called factor knots) on the same string. We find that the writhe (related to the handedness of crossing points) of composite knots is the sum of that of the ideal forms of the factor knots. By comparing ideal composite knots with simulated configurations of knotted, thermally fluctuating DNA, we conclude that the additivity of writhe applies also to randomly distorted configurations of composite knots and their corresponding factor knots. We show that composite knots with several factor knots may possess distinct structural isomers that can be interconverted only by loosening the knot.

The ideal representation of a knot corresponds to the shortest axial path of an impenetrable tube of uniform thickness forming a given knot type ${ }^{1}$. In other words, the ideal form of a knot is the spatial arrangement of the knotted tube with uniform thickness for which the length-to-diameter ratio is the smallest. These configurations can be obtained in computer simulations that maximize the thickness of knotted tubes while maintaining a fixed contour length. In the case of prime knots, computer runs end (in most cases); in the same final configuration, irrespective of the starting configuration of a given $\operatorname{knot}^{1}$. For composite knots, especially those involving asymmetric factor knots, the simulations frequently end in local minima. To find the global minimum of the length-todiameter ratio, it is necessary to carry out many independent simulations, testing different ways of combining two factor knots. For example, in searching for the ideal configuration of a composite knot composed of $6_{1}$ and $3_{1}$ factor knots, we created initial configurations where the symmetric $3_{1}$ knot was attached to different loops of the asymmetric $6_{1}$ knot. (The designations of knots follow the standard notation; the first number indicates the minimal number of crossings for a given knot and the subscript denotes the position of the knot among all other knots with the same crossing number ${ }^{3}$.) When both factor knots are symmetric, for example, $3_{1}$ or $4_{1}$, all loops are equivalent and there is no need to test many initial configurations. For different joinings of factor knots, the simulations frequently end in visually distinct configurations with similar length-to-diameter ratios, whereas simulations started from a given type of joining usually result in the same kinds of configurations with nearly identical length to diameter ratios. Although we are not sure if we have found the configurations with the smallest possible length-to-diameter ratios for the composite knots analysed, for the purpose of this Letter we designate as ideal the best configurations of the different knots that we have obtained in computer simulations. Figure 1 thus shows the ideal configurations of 16 composite knots where the factor knots range from trefoils up to six-noded knots. 


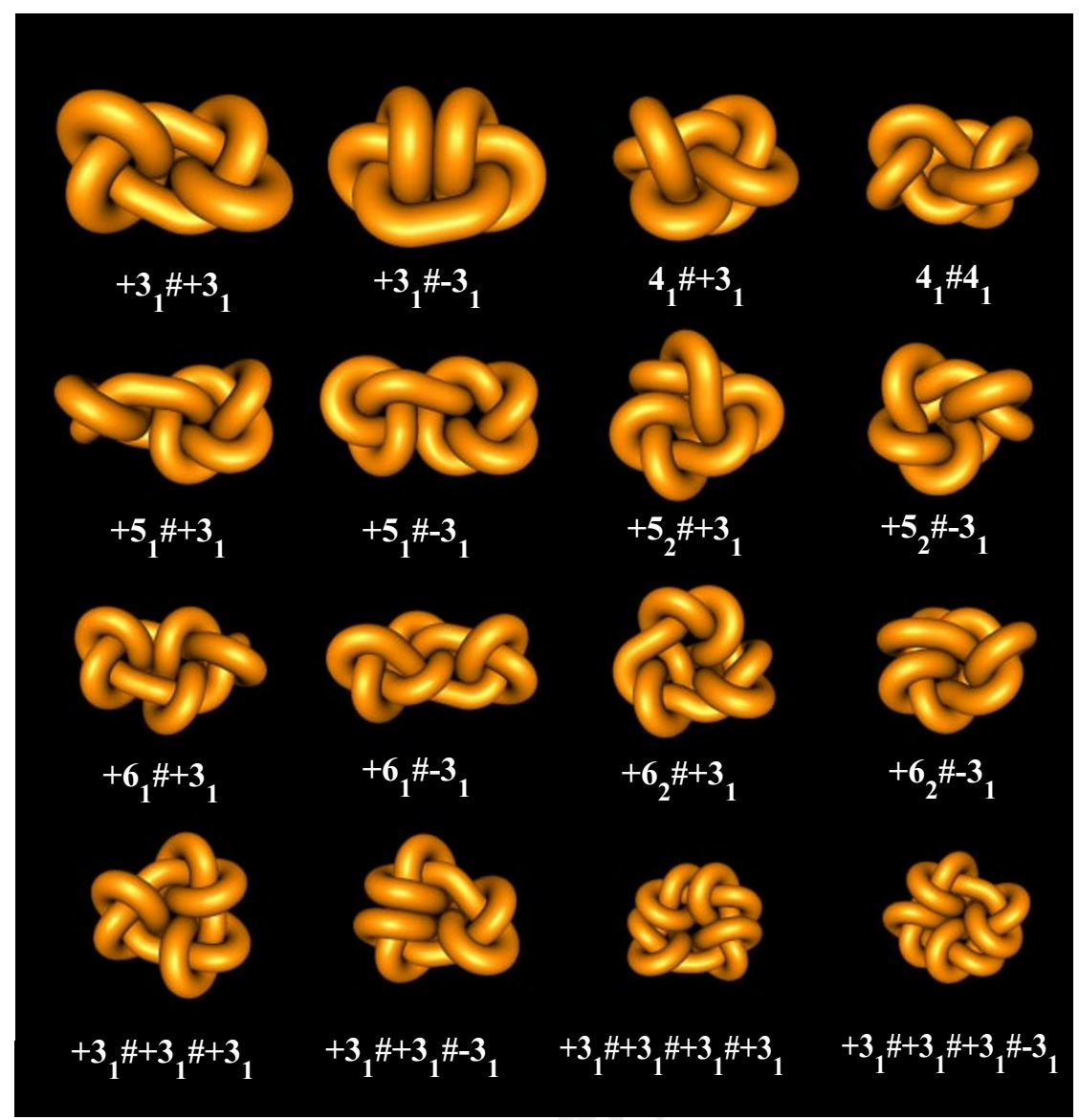

Figure 1 Ideal geometrical representations of several kinds of composite knots as viewed down their longest principal axes of inertia. (Three-dimensional models of these knots in VRML format are available at http:// rutchem.rutgers.edu/ seva/composite.html). The designations of the different composite knot types correspond to the factor knots that comprise them. For example, the designation $+3_{1} \# 4_{1}$ denotes a composite knot built from a right-handed trefoil and an achiral four-noded knot. We use the designations for factor knots given in standard tables of knots $^{3}$. To indicate the chirality of a factor knot, we introduce a + or - sign, respectively, for right- or lefthanded types. The absence of a sign indicates that the given factor knot is achiral. All ideal knot configurations shown here have the same axial length. As a result, the ratio between the contour lengths and diameters of the tubes making up the ideal knots increases progressively as the configurations become more complicated'. The optimized configurations are the result of a joint effort of two simulation programs based on two independent algorithms. The first one ${ }^{9}-$ simpler and faster, but less precise-simulates a mechanical process in which a completely flexible, frictionless tube, forming a knot of a given type, is uniformly inflated. Configurations found with this procedure are used as the starting configurations for the Metropolis-Monte Carlo simulations performed with the second program?

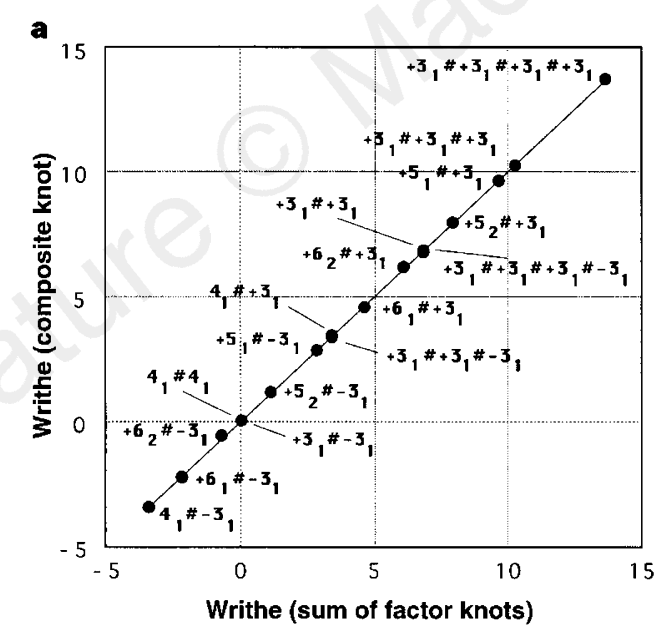

Figure 2 Additivity of the writhe and subadditivity of the relative lengths of ideal representations of composite knots. a, The 1:1 correspondence between the writhes of the ideal representations of different composite knots and the sums of the writhes of the respective factor knots in their ideal configurations. $\mathbf{b}$, The relative length of the composite knots is always smaller (by nearly a constant value) than the sum of the relative lengths of the factor knots constituting a given composite knot. The dashed line denotes a hypothetical situation where the relative lengths of the composite knots correspond to the sums of the relative lengths of the factor knots. Composite knots with three and four factor knots show correspondingly bigger deficits of the relative length.

b

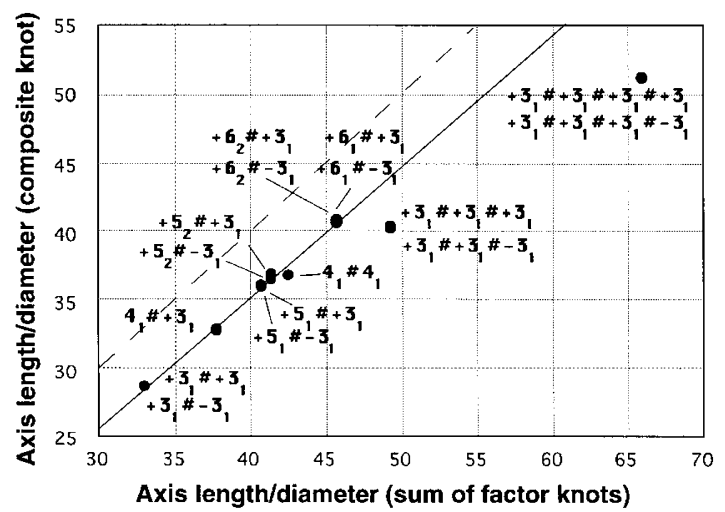




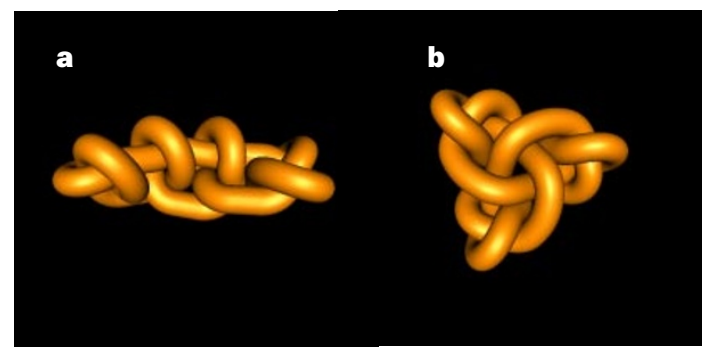

Figure 3 Linear (a) and star-shaped (b) configurations of composite $+3_{1} \#+3_{1} \#+3_{1}$ $\#+3$ knot.

To characterize the geometry of the ideal representations of the knots, it is informative to calculate their writhe, an accepted measure of the handedness of a curve in space ${ }^{4}$. One way to measure the writhe of a given knot is to observe the knot from many uniformly spread out directions on the unit sphere and to count the average number of right- and left-handed crossings per projection ${ }^{4}$. Right-handed crossings are scored as +1 and lefthanded ones as -1 . For example, the writhe of an ideal righthanded trefoil knot is close to +3.4 (ref. 1), a number which indicates that on average there are 3.4 more right-handed than left-handed crossings found when observing the knot from random directions. The data presented in Fig. 2a demonstrate that the writhe of the ideal configuration of a composite knot is equal to the sum of the writhe values of the ideal configurations of the constituent factor knots. The observed additivity of writhe is somewhat surprising because in general the writhe of a space curve is not additive. That is, if one arbitrarily joins the two complicated curves in space by removing parts of the original curves and replacing them with other segments, the writhe of the new curve is usually different from the sum of the writhes of the two original curves ${ }^{5}$. But in the ideal configurations of composite knots, even when the factor knots change slightly from the shapes that they assume as independent knots, these changes are self-compensatory and the writhe remains the same.

We have checked whether the mean writhe of the pool of thermally distorted polymer chains closed into different composite knots attains the same value as the writhe of the corresponding ideal composite knot. Taking advantage of our experience in modelling configurational ensembles of DNA knots under equilibrium conditions in solution ${ }^{1}$, we have used a Metropolis-Monte Carlo procedure to simulate millions of thermally distorted configurations of nicked DNA molecules closed in a given composite knot. As in our previous study, we have simulated knotted molecules of 5,400 base pairs, corresponding to $18 \mathrm{Kuhn}$ statistical segments (a size range typical of bacterial plasmid DNA). During the simulation the DNA chains are permitted to relax any torsional stress as would be the case for DNA containing interruptions in one of the strands of the double helix. Table 1 shows that the mean writhes of the Boltzmann populations of polymers forming different composite knots are practically equal to the writhes of the ideal configurations of these knots. We have shown ${ }^{1}$ that the mean writhe of a Boltzmann population of polymers forming a given prime knot is equal to the writhe of the ideal configuration of the knot; taking this into account, the data in Table 1 show that the additivity of writhe applies also to the time-averaged writhe values of thermally distorted configurations of the factor knots making up a given composite knot. Of course, in individual configurations of thermally distorted polymeric chains, the writhe of the composite knots varies. Random thermal distortions, however, are equally likely (for reasons of symmetry) to introduce regions of left- or right-handed winding in the knots. Thus, on average, the writhes of random knots, either created by thermal agitation or generated on a cubic
Table 1 Writhe of ideal and random configurations of composite knots

\begin{tabular}{|c|c|c|}
\hline Composite knot type & Writhe of ideal form & $\begin{array}{l}\text { Mean writhe of the } \\
\text { population of knotted DNA } \\
\text { molecules in solution }\end{array}$ \\
\hline$+3.1 \#+3.1$ & 6.82 & 6.78 \\
\hline$+3.1 \#-3.1$ & 0.00 & 0.00 \\
\hline $4.1 \#+3.1$ & 3.39 & 3.42 \\
\hline$+5.1 \#+3.1$ & 9.67 & 9.61 \\
\hline$+5.1 \#-3.1$ & 2.84 & 3.00 \\
\hline$+5.2 \#+3.1$ & 7.98 & 7.96 \\
\hline$+5.2 \#-3.1$ & 1.17 & 1.14 \\
\hline $4.1 \# 4.1$ & 0.05 & 0.03 \\
\hline$+6.1 \#+3.1$ & 4.55 & 4.40 \\
\hline$+6.1 \#-3.1$ & -2.25 & -2.15 \\
\hline$+6.2 \#+3.1$ & 6.15 & 6.19 \\
\hline$+6.2 \#-3.1$ & -0.55 & -0.54 \\
\hline
\end{tabular}

We note the good correspondence between the mean writhes of the simulated Boltzmann populations of randomly distorted, nicked DNA molecules forming different composite knots and the writhe values of the corresponding ideal configurations of the composite knots. Simulations of equilibrium configurations of composite and prime knots were performed for DNA molecules (5,400 base pairs long) in low salt solution. Taking into account statistical errors in sampling the configurational space of randomly distorted knots and the error in calculating the writhe with the Gauss integra $\left.\right|^{4,7}$, the data in the second and third columns can be regarded as equal. See ref. 8 for further details on the simulation procedure.

lattice ${ }^{6}$, correspond to the writhe of the ideal representation of a given knot.

The principal characteristic of an ideal knot is the ratio of its length to diameter, which we call the relative length. This ratio is a good measure of the complexity of a knot; the higher the relative length is, the more complicated the knot is. Although the relative lengths of the constituent ideal factor knots do not sum to that of their ideal composite knot, the total exceeds the relative length of the composite knot by roughly the same value regardless of the type of composite knot; see Fig. 2b. Closer inspection of Fig. 1 reveals the reason for this subadditivity. If we assume that the diameter of the tube forming the composite knots shown in the figure is $1 \mathrm{~cm}$, it should not be too difficult to see that in the formed composite knots each factor knot loses one half of a quasi-circular arc of $1 \mathrm{~cm}$ radius and gains one straight segment of $\sim 1 \mathrm{~cm}$ length. The arcs which are eliminated by the fusion are, in fact, somewhat longer than $\pi$, as the winding angles are not equal to $90^{\circ}$. Thus, in total, the length decrease is usually somewhat greater than the diameter multiplied by $2 \pi-2$. Because the junction points of factor knots can have different local structures for various types of composite knots (see Fig. 1), the lengths of eliminated arcs and new linking segments may vary. This produces small deviations from subadditivity with a constant difference.

As already mentioned, the loops of symmetric factor knots such as $3_{1}$ or $4_{1}$ are identical. Thus, irrespective of which loop is used to fuse two such knots, no major reshuffling of the knot is required to reach the ideal configurations of $3_{1} \# 3_{1}, 3_{1} \# 4_{1}$ or $4_{1} \# 4_{1}$ composite knots (see Fig. 1 for details of this nomenclature). However, there are several ways of arranging composite knots constructed from multiple repetitions of $3_{1}$ or $4_{1}$ factor knots, and a major reshuffling of the whole length of the composite knot is needed to convert from one form into another. Consider the composite knot formed from four $3_{1}$ knots. The factor knots can be joined circularly (Fig. 1, bottom row), linearly (Fig. 3a) or in a star-like configuration with the loops of a central trefoil linked to the three other trefoils (Fig. $3 \mathrm{~b}$ ). To change from the linear to the star-shaped configuration of a composite knot, energy is required to loosen the knot. By providing more length or by shrinking the diameter, the factor knots can be rearranged in such a way that a different isomer of the composite knot will appear. Composite knots made up of multiple repeats of four-noded knots recall hydrocarbon molecules, as the ideal form of 
the four-noded knot has roughly tetrahedral symmetry, and it is possible to create star-shaped arrangements where the central fournoded knot coordinates to four other knots.

Received 19 March; accepted 13 May 1997

1. Katritch, V. et al. Geometry and physics of knots. Nature 384, 142-145 (1996)

2. Stasiak, A., Katritch, V., Bednar, J., Michoud, D. \& Dubochet, J. Electrophoretic mobility of DNA knots. Nature 384, 122 (1996).

3. Rolfsen, D. Knots and Links (Publish or Perish, Berkeley, CA, 1976).

4. Fuller, F. B. The writhing number of a space curve. Proc. Natl Acad. Sci. USA 68, 815-819 (1971).

5. Fuller, F. B. Decomposition of the linking number of a closed ribbon: a problem from molecular biology. Proc. Natl Acad. Sci. USA 75, 3557-3561 (1978)

6. Janse van Rensburg, E. J., Orlandini, E., Sumners, D. W., Tesi, M. C. \& Whittington, S. G. The writhe of knots in the cubic lattice. J. Knot Theory Ramif. 6, 31-44 (1997).

7. Calugareanu, G. L'intégral de Gauss et l'analyse des noeuds tridimensionnels. Rev. Math. Pur. Appl. 4 5-20 (1959)

8. Vologodskii, A. V., Levene, S. D., Klenin, K. V., Frank-Kamenetskii, M. \& Cozzarelli, N. R. Conformational and thermodynamic properties of supercoiled DNA. J. Mol. Biol. 227, 1224-1243 (1992).

9. Pieranski, P. Search of ideal knots. ProDialog 5, 111-120 (1996).

Acknowledgements. This work was supported by the US Public Health Service, the Polish Committee of Scientific Research, the Swiss National Foundation and by Foundation Herbette, University of Lausanne. We thank R. Scharein for making available his KnotPlot program.

Correspondence should be addressed to A.S. (e-mail: Andrzej.Stasiak@lau.unil.ch).

\section{The dynamics of partially extended single molecules of DNA}

\section{Stephen R. Quake ${ }^{\star}$, Hazen Babcock \& Steven Chu}

Physics Department, Stanford University, Stanford, California 94305, USA

The behaviour of an isolated polymer floating in a solvent forms the basis of our understanding of polymer dynamics ${ }^{1,2}$. Classical theories describe the motion of a polymer with linear equations of motion, which yield a set of 'normal modes', analogous to the fundamental frequency and the harmonics of a vibrating violin string. But hydrodynamic interactions make polymer dynamics inherently nonlinear, and the linearizing approximations required for the normal-mode picture have therefore been questioned $^{1}$. Here we test the normal-mode theory by measuring the fluctuations of single molecules of DNA held in a partially extended state with optical tweezers. We find that the motion of the DNA can be described by linearly independent normal modes, and we have experimentally determined the eigenstates of the system. Furthermore, we show that the spectrum of relaxation times obeys a power law.

The starting point for describing the motion of a polymer is the Rouse model of beads interconnected with gaussian springs. If the interactions between different segments (beads) of the polymer $R_{n}$ and $R_{m}$ ( $n$ and $m$ denote the order of the beads along the length of the polymer) are localized to nearest neighbours, each of the segments $R_{n}$ satisfies a linear differential equation decoupled from the other segments. This linear system allows one to describe the dynamics in terms of a set of normal modes with mode amplitudes $X_{p}$ defined as $X_{p}=(1 / N) \int_{0}^{N} R_{n} \cos (\pi p n / N) \mathrm{d} n$ (where $p$ is an integer denoting the $p$ th mode and $N$ is the total number of beads). Associated with the normal modes is a set of relaxation times, $\tau_{p}$, which describe the decay in the time correlations of the normalmode amplitudes $\left\langle X_{p}(t) X_{q}(0)\right\rangle \sim \delta_{p q} e^{-t / \tau_{p}}$ (where $q$ is the $q$ th mode).

Hydrodynamic interactions between segments of the polymer were first included by Zimm ${ }^{3}$ by assuming the surrounding fluid is incompressible and obeys the Navier-Stokes equation (known as the Kirkwood approximation ${ }^{4}$ ). The resulting coupling of each polymer segment $R_{n}$ to all of the other segments $R_{m}$ leads to a

* Present address: Department of Applied Physics, Caltech, MS 128-95, Pasadena, California 91125, USA. nonlinear set of differential equations as the coupling depends on the instantaneous configuration of the polymer. These equations can be linearized by first averaging the distance $\left|R_{m}-R_{m}\right|$ between segments over the distribution of accessible configurations. This socalled 'pre-averaging' approximation linearizes the set of coupled differential equations and allows one to construct a set of normal modes similar to the Rouse modes.

It has been argued that a normal-mode description of a polymer can be only a rough approximation of the true dynamics and may not have any fundamental validity ${ }^{1}$. Apart from questioning the soundness of the pre-averaging approximation, other nonlinear effects such as excluded-volume effects, forbidden crossings, and knots were not included in Zimm's treatment. These and other nonlinear effects have led de Gennes ${ }^{1}$ and others to emphasize dynamical scaling laws rather than a normal-mode description. Scaling laws link relaxation rates to the spatial scale of interest, but do not require knowledge of the precise shape of the relaxation spectrum. On the other hand, the normal-mode concept requires that the relaxation spectrum be described by a set of discrete frequencies.

The analysis of the relaxation of polymers perturbed from equlibrium can yield a frequency spectrum by taking the inverse Laplace transform of the relaxation spectrum, but these methods do not provide an unambiguous decomposition into discrete frequencies ${ }^{5,6}$. Dynamic light-scattering experiments ${ }^{7,8}$, viscoelastic and oscillatory flow birefringence studies ${ }^{9-12}$ and transient electric birefringence experiments ${ }^{13,14}$ probe relaxation rates at size scales considerably smaller than the overall coil size, but these measurements represent an average over what could be a continuous spectrum of relaxation rates. Dielectric relaxation of dipole-inverted cis-polyisoprene has been used to measure the first two normalmode eigenfunctions, and a tentative extrapolation was used to derive the third ${ }^{15-17}$. However, this method does not measure a relaxation mode spectrum, and does not discriminate between linear and nonlinear effects.

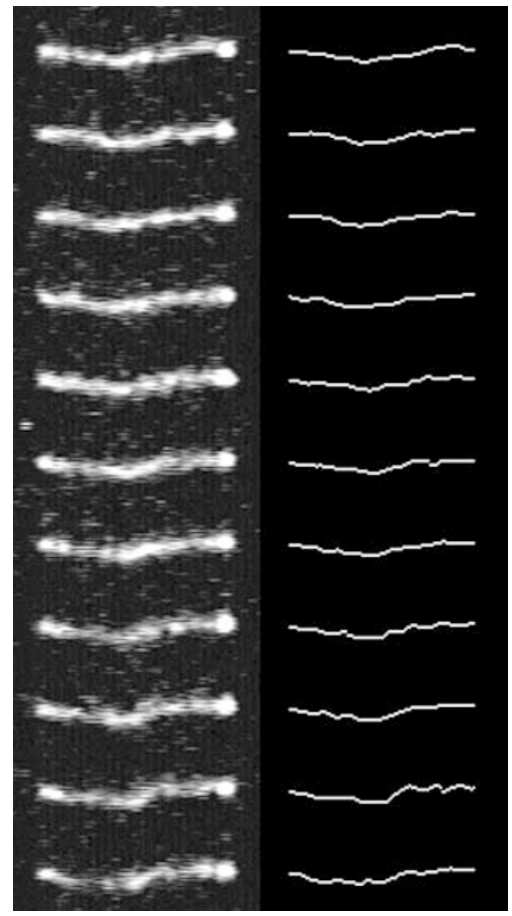

Figure 1 Video images of single molecules of DNA held at an extension of $12 \mu \mathrm{m}$. The column on the left shows a series of successive raw video images; the column on the right shows the corresponding parametrizations used in the data analysis. 